\title{
THE IDENTIFICATION OF LINEAR AND NON-LINEAR MODELS OF A TURBOCHARGED AUTOMOTIVE DIESEL ENGINE
}

\author{
S. A. Billings and S. Chen \\ Department of Control Engineering, University of Sheffield, Sheffield, U.K. \\ AND \\ R. J. BACKHOUSE $\dagger$ \\ Department of Mechanical Engineering, UMIST, Manchester, U.K.
}

(Received February 1988, accepted September 1988)

\begin{abstract}
The identification results obtained from a study on a Leyland TL11 turbocharged, direct injection diesel engine are presented. Two sets of data corresponding to low and high engine speed tests, which were recorded from experimental trials on the engine, are analysed. The identification of both linear and non-linear difference equation models are described to represent the relationship between the fuel rack position (input) and the engine speed (output).
\end{abstract}

\section{INTRODUCTION}

This paper describes the identification of both linear and non-linear models of a Leyland TL11 6 cylinder $11 \cdot 1$ litre diesel engine with Holset VH2C turbocharger. The data was collected from an engine at Bath University by Richard Backhouse (UMIST) and models were subsequently identified using the identification techniques developed in the Department of Control Engineering, University of Sheffield. Two sets of data are used in the identification and in both cases the input is fuel rack position and the output is engine speed. The first data set was obtained from a low engine speed test at a sample rate of $80 \mathrm{msec}$ while the second data set was recorded from a high engine speed test with a sample rate of $100 \mathrm{msec}$. The disparity in the sampling rate meant that two different models were necessary one for each experimental condition. The analysis of the first data set required the estimation of a non-linear model whereas the second data set could be adequately described by a linear model.

The paper begins with a brief description of the identification technique which was used in the analysis. The technique can determine the structure or which terms to include in the model and can provide optimal final parameter estimates in an efficient manner. This is followed by a description of the two data sets. Finally, the identification of linear and non-linear models to describe the engine are presented.

\section{AN EFFICIENT IDENTIFICATION TECHNIQUE FOR NON-LINEAR SYSTEMS}

\subsection{A CLASS OF NARMAX MODELS}

An input-output model is a means for representing the input-output relationship of a system and therefore choosing a suitable model set is an important step in system

$\dagger$ Present address: Napier Turbochargers Ltd, Lincoln, U.K. 
identification. The NARMAX (non-linear ARMAX) model [7,13] provides a unified representation for a wide class of discrete-time non-linear stochastiic control systems. A general NARMAX model takes the following form

$$
y(t)=f\left(y(t-1), \ldots, y\left(t-n_{y}\right), u(t-1), \ldots, u\left(t-n_{u}\right), e(t-1), \ldots, e\left(t-n_{e}\right)\right)+e(t)
$$

where $\bar{y}(t), u(t)$ and $e(t)$ are the system output, input and noise respectively; $n_{y}, n_{u}$ and $n_{e}$ are the orders of the output, input and noise; $\{e(t)\}$ is assumed to be a white sequence; and $f(\cdot)$ is some non-linear function.

In order to use model (1) as a basis for identification, a means of parametrisation is required and a polynomial expansion of $f(\cdot)$ is a convenient but by no means the only choice. Expanding $f(\cdot)$ as a polynomial of degree $L$ gives the representation

$$
y(t)=\sum_{i=1}^{n} \theta_{i} x_{i}(t)+e(t)
$$

where

$$
n=\sum_{i=0}^{L} n_{i} ; n_{0}=1, n_{i}=n_{i-1}\left(n_{y}+n_{u}+n_{e}+i-1\right) / i, i=1, \ldots, L
$$

and

$$
\left.\begin{array}{c}
x_{1}(t)=1 \\
x_{i}(t)=\prod_{j=1}^{p} y\left(t-n_{y j}\right) \cdot \prod_{k=1}^{q} u\left(t-n_{u k}\right) \cdot \prod_{m=1}^{r} e\left(t-n_{e m}\right) \\
i=2, \ldots, n, \quad p, q, r \geqslant 0, \quad 1 \leqslant p+q+r \leqslant L, \\
1 \leqslant n_{y j} \leqslant n_{y}, \quad 1 \leqslant n_{u k} \leqslant n_{u}, \quad 1 \leqslant n_{e m} \leqslant n_{e}
\end{array}\right\}
$$

By convention, $p=0$ indicates that $x_{i}(t)$ contains no $y(\cdot)$ terms. Similarly, $q=0$ indicates that $x_{i}(t)$ contains no $u(\cdot)$ terms and $r=0$ indicates that $x_{i}(t)$ contains no $e(\cdot)$ terms. Regrouping terms in equation (2) yields

$$
\begin{aligned}
y(t)= & f^{p}\left(y(t-1), \ldots, y\left(t-n_{y}\right), u(t-1), \ldots, u\left(t-n_{u}\right)\right) \\
& +f^{n}\left(y(t-1), \ldots, y\left(t-n_{y}\right), u(t-1), \ldots, u\left(t-n_{u}\right), e(t-1), \ldots, e\left(t-n_{e}\right)\right)+e(t)
\end{aligned}
$$

where $f^{p}(\cdot)$ contains all terms $\theta_{i} x_{i}(t)$ with $r=0$ and $f^{n}(\cdot)$ contains all terms $\theta_{i} x_{i}(t)$ with $r \neq 0 . f^{p}(\cdot)$ is referred to as the process model and $f^{n}(\cdot)$ as the noise model. An important case of model (5) is

$$
y(t)=f^{p}\left(y(t-1), \ldots, y\left(t-n_{y}\right), u(t-1), \ldots, u\left(t-n_{u}\right)\right)+\sum_{i=1}^{n_{e}} c_{i} e(t-i)+e(t)
$$

where the noise model $f^{n}(\cdot)$ is linear. The identification of several industrial systems has shown that many can be modelled in the form of equation (6). Some examples are a $6996 \mathrm{bhp}$ industrial diesel generator [5], a liquid level system [2] and a heat exchanger $[4,17]$. It is obvious that the linear ARMAX model

$$
y(t)=\sum_{i=1}^{n_{y}} a_{i} y(t-i)+\sum_{i=1}^{n_{4}} b_{i} u(t-i)+\sum_{i=1}^{n_{e}} c_{i} e(t-i)+e(t)
$$

is a simple case of equation (6). 


\subsection{THE IDENTIFICATION TECHNIQUE}

When the system is non-linear, direct estimation based on equation (2) may involve an excessive number of terms. Simply increasing the orders $n_{y}, n_{u}$ and $n_{e}$, and the degree $L$ of the polynomial expansion to achieve the desired accuracy will in general result in an excessively complex model and possibly numerical ill-conditioning. The determination of the structure or which terms to include in the model is therefore essential if a parsimonious model is to be determined from the large number of candidate terms equation (3). An orthogonal regression estimator $[6,12]$ has been developed which can select a subset of significant terms very efficiently. The basic idea is to transfer equation (2) into an equivalent orthogonal equation. Because of the orthogonal property, significant terms can be determined in a particularly simple forward regression procedure [6]. The criterion of selecting terms is according to how large a proportion of the output variance that a term can explain. The estimated values of the parameters selected are also provided. Details of this method have been given in the two references mentioned above and will not be repeated here.

An advantage of the orthogonal estimator is that significant parameters can be determined recursively and quite independently of the other terms already selected. Furthermore, the estimation of the process and noise model parameters can be decoupled [12]. This is particularly useful for the identification of model (5). A parsimonious process model is first determined. This model will not be affected by whatever noise model is produced later. The initial residuals are computed based on this process model and a noise model can be selected. Then a revised residual sequence is calculated and an improved noise model is determined. A few iterations are often enough to find a final parsimonious noise model. At this stage, a prediction-error estimator $[3,16]$ can be used to produce the final optimal parameter estimates. Alternative methods of structure detection are available $[9,11,14]$ and these can either be used instead of the orthogonal estimator or in conjuction with it to investigate reducing the number of parameters further.

For model (6), a simplified procedure can be applied. After a parsimonious process model has been determined using the orthogonal estimator, a value of $n_{e}$ is chosen and the prediction-error estimator is called to provide optimal parameter estimates. In the case of a linear model equation (7), the determination of the orders $n_{y}$ and $n_{u}$ becomes a very simple matter using the orthogonal estimator as will be shown later.

\subsection{MODEL VALIDITY TESTS}

Let $\hat{f}(\cdot)$ be an estimate of $f(\cdot)$. Then the one-step ahead prediction of $y(t)$ is defined as

$$
\hat{y}(t)=\hat{f}\left(y(t-1), \ldots, y\left(t-n_{y}\right), u(t-1), \ldots, u\left(t-n_{u}\right), \varepsilon(t-1), \ldots, \varepsilon\left(t-n_{e}\right)\right)
$$

where $\varepsilon(t)$ is the residual or prediction error and is given by

$$
\varepsilon(t)=y(t)-\hat{y}(t) \text {. }
$$

The model predicted output is defined as

$$
\hat{y}_{d}(t)=\hat{f}\left(\hat{y}_{d}(t-1), \ldots, \hat{y}_{d}\left(t-n_{y}\right), u(t-1), \ldots, u\left(t-n_{u}\right), 0, \ldots, 0\right)
$$

and the deterministic prediction error is given by

$$
\varepsilon_{d}(t)=y(t)-\hat{y}_{d}(t) .
$$

A display of $y(t), \hat{y}(t), \varepsilon(t), \varepsilon_{d}(t)$ often gives some preliminary information regarding the goodness of fit of the estimated model. 
If the identified model is adequate, the prediction errors should satisfy the following conditions $[3,8]$

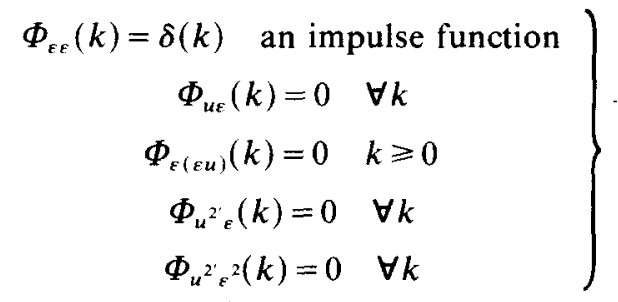

where $\Phi_{x y}(k)$ indicates the cross correlation function between $x(t)$ and $y(t), u^{2}(t)=$ $u^{2}(t)-\overline{u^{2}(t)}$ and $\overline{u^{2}(t)}$ represents the time average or mean value of $u^{2}(t)$. Therefore, if at least one of the correlation functions has values well outside the confidence limits, the model is certainly inadequate or biased. Experience has shown that if these tests are used in conjunction with the estimation algorithm, the experimenter can often infer a great deal of information regarding deficiencies in the fitted model. Indeed the tests equation (12) often indicate which terms should be included in the model to improve the fit.

Alternatively, a statistical test known as the chi-square test $[3,10,14]$ can be used to validate an estimated model. The value of the chi-square statistic is calculating using the formula

$$
\eta=N \mu^{\tau}\left(\Gamma^{\tau} \Gamma\right)^{-1} \mu
$$

where $N$ is the data length and

$$
\begin{gathered}
\mu=\frac{1}{N} \sum_{t=1}^{N} \frac{Z(t) \varepsilon(t)}{\sigma} \\
\sigma^{2}=E\left[\varepsilon^{2}(t) \mid x^{t}\right] \\
x^{t}=\left[\begin{array}{c}
y^{t-1} \\
\varepsilon^{t-1} \\
u^{t}
\end{array}\right]=(y(t-1), \ldots, y(1), \varepsilon(t-1), \ldots, \varepsilon(1), u(t), \ldots, u(1))^{\tau} \\
Z(t)=Z\left(x^{t}\right) \\
\Gamma^{\tau} \Gamma=\frac{1}{N} \sum_{t=1}^{N} Z(t) Z^{\tau}(t) .
\end{gathered}
$$

A convenient choice of the vector function $Z(t)$ is

$$
Z(t)=(m(t), m(t-1), \ldots, m(t-s+1))^{\tau}
$$

where $m(t)$ is a monomial of $x^{t}$, for instance it could be selected as

$$
\begin{aligned}
m(t)= & y^{i_{11}}(t-1) y^{i_{22}}(t-2) y^{i_{33}}(t-3) u^{i_{11}}(t-1) u^{i_{12}}(t-2) \\
& \times u^{i_{u 3}}(t-3) \varepsilon^{i_{\varepsilon 1}}(t-1) \varepsilon^{i_{\varepsilon 2}}(t-2) \varepsilon^{i_{\varepsilon 3}}(t-3)
\end{aligned}
$$

where $i_{y 1}, i_{y 2}, i_{y 3}, i_{u 1}, i_{u 2}, i_{u 3}, i_{\varepsilon 1}, i_{\varepsilon 2}, i_{\varepsilon 3} \geqslant 0$ and $i_{y 1}+i_{y 2}+i_{y 3}+i_{u 1}+i_{u 2}+i_{u 3}+i_{\varepsilon 1}+i_{\varepsilon 2}+i_{\varepsilon 3}>$ 0 . Under the null hypothesis that the data are generated by the model, the statistic $\eta$ is an asymptotically chi-squared distribution with $s$ degrees of freedom. Thus, if the values of $\eta$ for several different types of $m(t)$ are within the acceptance region (95\%), that is,

$$
\eta<k_{\alpha}(s)
$$

the model can be regarded as an adequate one, where $k_{\alpha}(s)$ is the critical value of the chi-square distribution with $s$ degrees of freedom and a given level of significance $\alpha(0 \cdot 05)$. 


\section{IDENTIFICATION OF THE DIESEL ENGINE}

\subsection{THE DATA SETS}

In this section, the engine details and experimental conditions are briefly described.

Engine: Leyland TL11, turbocharged, direct injection diesel engine

Configuration: 6 cylinders in-line, 4-stroke

Turbocharger: Holset VH2C

Bore and stroke: $127 \times 146.05 \mathrm{~mm}$

Swept volume: $11 \cdot 1$ litres

Rating: $190 \mathrm{~kW}$ at $2100 \mathrm{rpm}$

Dynamometer: Lucas swash-plate hydrostatic pump.

A hydraulic (position) actuator acts directly onto the fuel rack of the injection pump. The actuator (i.e., rack) position is controlled using feedback from a linear position transducer. The actuator with feedback and servo valve acts as a pure time delay of approximate $23 \mathrm{~ms}$ for excitation signals up to $10 \mathrm{~Hz}$. The transducer output range is 0 to $+10 \mathrm{~V}$. The rack has a $3 \mathrm{~mm}$ dead band so that fuel injection does not commence until the output voltage reaches approximately $1 \cdot 35 \mathrm{~V}$. The maximum fuel injection rate of $137 \mathrm{~mm}^{3} /$ stroke is reached at $1500 \mathrm{rpm}$. Above this speed the fuelling falls steadily to approximately $125 \mathrm{~mm}^{3} /$ stroke at maximum speed.

In the experimental trials, the following engine variables were measured: engine speed, boost pressure, exhaust temperature and smoke capacity. All the outputs are in the range 0 to $+10 \mathrm{~V}$ or 0 to $-10 \mathrm{~V}$. The hydrostatic pump used as dynamometer has a characteristic

$$
L_{b}=K \cdot N_{E}^{\alpha}
$$

where $L_{b}$ is the brake torque, $N_{E}$ is the engine speed, $K$ is a constant depending on swash-plate angle and hydrostatic oil pressure and $\alpha$ is a constant $(\approx 1.6)$.

Two tests covering different engine speed ranges were performed. In order to achieve a near full load torque in each test it was necessary to vary the dynamometer constant $K$ between the two tests. Within each test $K$ remained constant so that the load was proportional to $N_{E}^{1.6}$. In each case the fuel rack was perturbed by a square wave signal of large amplitude with a PRBS superimposed. The PRBS was a 31-bit sequence (from a 5-bit generator) and approximately coincided with the half period of the square wave. Once the engine had reached its stable operating condition the rack demand was switched to the square wave plus PRBS signal and the input and output variables were recorded on a uv recorder over about ten complete cycles of the input sequence. Notice that the design of inputs for non-linear systems is much more complex than the linear case [15]. Details of the two experimental tests are summarised in Table 1.

Boost pressure, exhaust temperature and smoke opacity are intermediate variables. In the present study, only the relationship between the fuel rack position and engine speed is investigated. The two raw data sets obtained in the low and high speed tests are supplied as files b6shv2.dta and b6shv6.dta respectively, where the input $u(t)$ is fuel rack position and the output $y(t)$ is engine speed. These are illustrated on Fig. 1. All the data were digitised by hand from the uv recordings; because the sample point was taken to be at the end of the relevant bit period, terms in $u(t)$ are likely to appear in the model. This is unlike most discrete-time systems where the influence of $u(t)$ on the output appears at least one sampling interval later as shown in equation (1). In order to apply the identification package written for the model (1) to the data, the input sequence in the original data is moved one-step forward, that is, $u(t)$ is replaced by $u(t+1)$. To reduce the possible effects of the initial conditions, the first six points were discarded from b6shv2.dta and the first 14 points were discarded from b6shv6.dta. These choices were 
TABle 1

Details of the two experimental tests

\begin{tabular}{lcc}
\hline & Low : peed test & High speed test \\
\hline PRBS bit period & $0 \cdot 100 \mathrm{sec}$ & $0 \cdot 080 \mathrm{sec}$ \\
PRBS repeating sequence & $31 \mathrm{bits}$ & $31 \mathrm{bits}$ \\
Square wave frequency & $0 \cdot 16 \mathrm{~Hz}$ & $0 \cdot 20 \mathrm{~Hz}$ \\
PRBS amplitude & $\pm 0 \cdot 4 \mathrm{~V}$ & $\pm 0 \cdot 4 \mathrm{~V}$ \\
Square wave amplitude (with bias) & $4 \cdot 77 \pm 0.65 \mathrm{~V}$ & $4 \cdot 5 \pm 0 \cdot 5 \mathrm{~V}$ \\
Engine speed range & $680-1180 \mathrm{rpm}$ & $1300-1830 \mathrm{rpm}$ \\
Boost pressure range (absolute) & $1 \cdot 098-1 \cdot 375 \mathrm{bar}$ & $1 \cdot 26-1 \cdot 59 \mathrm{bar}$ \\
Exhaust temperature range & $402-535^{\circ} \mathrm{C}$ & $552-570^{\circ} \mathrm{C}$ \\
Sampling interval & $80 \mathrm{msec}$ & $100 \mathrm{msec}$ \\
\hline
\end{tabular}
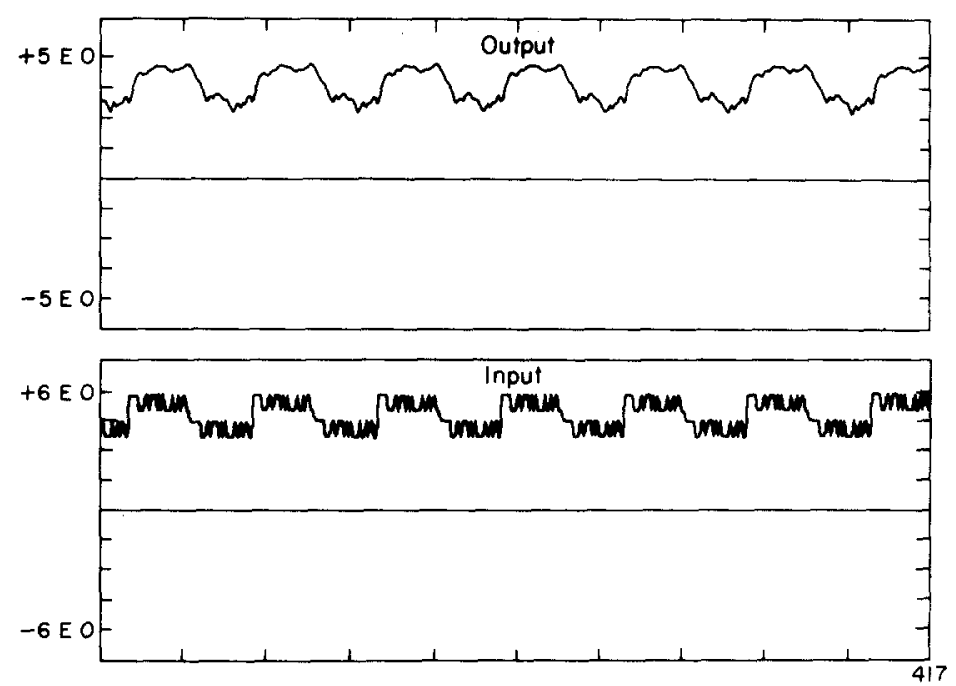

(a)
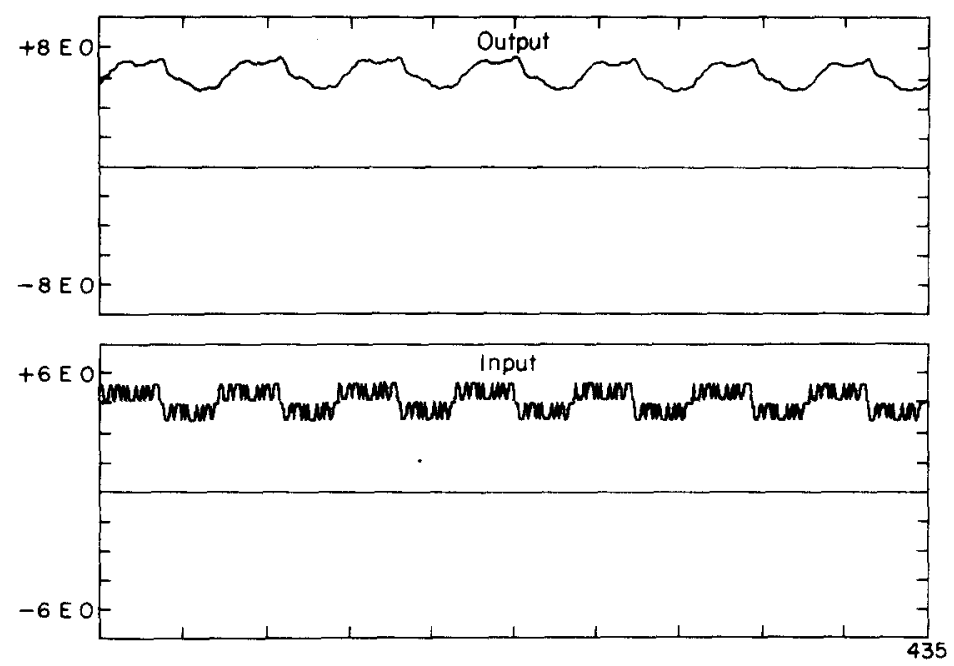

(b)

Figure 1. Raw data sets. (a) b6shv2.dta; (b) b6shv6.dta. 
made from a visual inspection of the data. The data sets obtained by the above two operations were renamed as mob6shv2.dta and mob6shv6.dta respectively. Models were fitted to these two modified data sets. Therefore terms containing $u(t-i), i>0$ obtained in the identification study below should be changed to terms containing $u(t+1-i)$ in the real model of the engine. Notice that because the data were sampled by hand, errors are likely to appear in synchronising the sampling times for $u(t)$ and $y(t)$, and relatively large noise components might therefore have been introduced into the data.

\subsection{LINEAR ANALYSIS OF THE LOW ENGINE SPEED CASE}

The first step in linear analysis of the data in mob6shv2.dta involved the application of the forward regression orthogonal estimator to determine the correct orders $n_{y}$ and $n_{u}$. This was achieved by setting $n_{u}$ and $n_{y}$ to some large value, $n_{u}=n_{y}=20$ in this case, and allowing the orthogonal estimator to search through and order the terms by measuring the contribution that each term makes to the output variance (e.r.r.). With a tolerance (unexplained output variance) of 0.0001 , the orthogonal estimator selected ten terms from the total of 41 possible terms (Table 2). Inspection of the e.r.r. results in Table 2 suggests that a model of the form

$$
y(t)=\text { constant }+a_{1} y(t-1)+b_{1} u(t-1)+b_{2} u(t-2)+\sum_{i=1}^{n_{e}} c_{i} e(t-i)+e(t)
$$

may provide an adequate representation for this data set. The prediction-error estimator was then used to provide optimal parameter estimates in equation (23). Table 3 gives the results for $n_{e}=0$ to $n_{e}=5$, and the corresponding correlation tests for $n_{e}=0,3$ and 5 are shown in Fig. 2.

TABLE 2

Orthogonal estimation of linear model (mob6shv2.dta)

\begin{tabular}{rcrrr}
\hline O.s. & Terms & \multicolumn{1}{c}{ Estimates } & \multicolumn{1}{c}{ E.r.r.s } & St.de.s \\
\hline 1 & $(2) y(t-1)$ & $0 \cdot 7186 E+0$ & $0.9989 E+0$ & $0.9283 E-2$ \\
2 & $(23) u(t-2)$ & $0 \cdot 1678 E+0$ & $0.6346 E-3$ & $0.4785 E-2$ \\
3 & $(22) u(t-1)$ & $0 \cdot 1262 E+0$ & $0 \cdot 1570 E-3$ & $0 \cdot 4045 E-2$ \\
4 & $(1)$ constant & $-0.4666 E+0$ & $0 \cdot 1460 E-3$ & $0 \cdot 2681 E-1$ \\
5 & $(14) y(t-13)$ & $0 \cdot 6822 E-1$ & $0 \cdot 2895 E-4$ & $0 \cdot 1087 E-1$ \\
6 & $(18) y(t-17)$ & $-0 \cdot 3722 E-1$ & $0 \cdot 9678 E-5$ & $0 \cdot 7084 E-2$ \\
7 & $(8) y(t-7)$ & $-0 \cdot 1189 E+0$ & $0 \cdot 4320 E-5$ & $0 \cdot 1804 E-1$ \\
8 & $(11) y(t-10)$ & $0 \cdot 6241 E-1$ & $0 \cdot 3735 E-5$ & $0 \cdot 1341 E-1$ \\
9 & $(6) y(t-5)$ & $0 \cdot 4246 E-1$ & $0 \cdot 2825 E-5$ & $0.1287 E-1$ \\
10 & $(30) u(t-9)$ & $0 \cdot 1863 E-1$ & $0 \cdot 2097 E-5$ & $0.6563 E-2$ \\
\hline
\end{tabular}

O.s., order of selection in regression procedure; E.r.r.s, error reduction ratios; St.de.s, standard deviations of estimates.

It is seen that when $n_{e}$ is increased to five, the correlation functions $\Phi_{\varepsilon \varepsilon}(k)$ and $\Phi_{\varepsilon(\varepsilon u)}(k)$ have improved slightly and are almost within the confidence limits but $\Phi_{u^{2}, \varepsilon^{2}}(k)$ has hardly changed. Notice that the dotted lines in Fig. 2 represent the approximate $95 \%$ confidence limits. In practice the confidence limits will bell out away from the axis for increasing $k$ so that the values of $\Phi_{\varepsilon \varepsilon}(k)$ and $\Phi_{\varepsilon(\varepsilon u)}(k)$ which are slightly outside the limits in Fig. 2 may in reality be acceptable. Various linear models with higher orders $n_{y}$, 


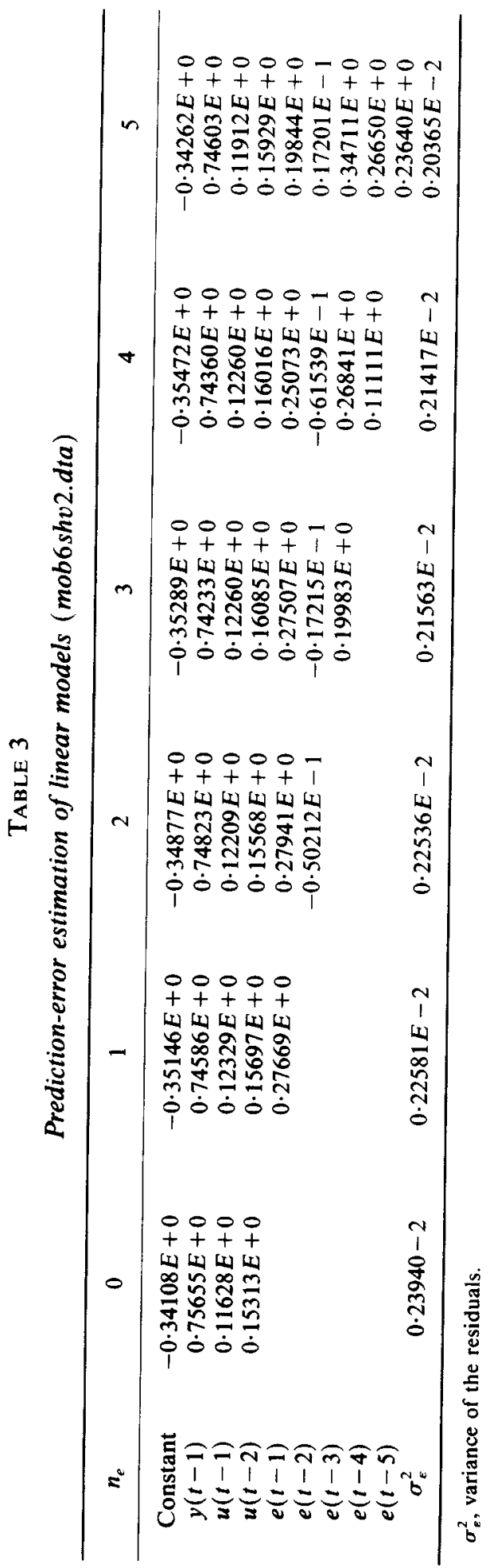



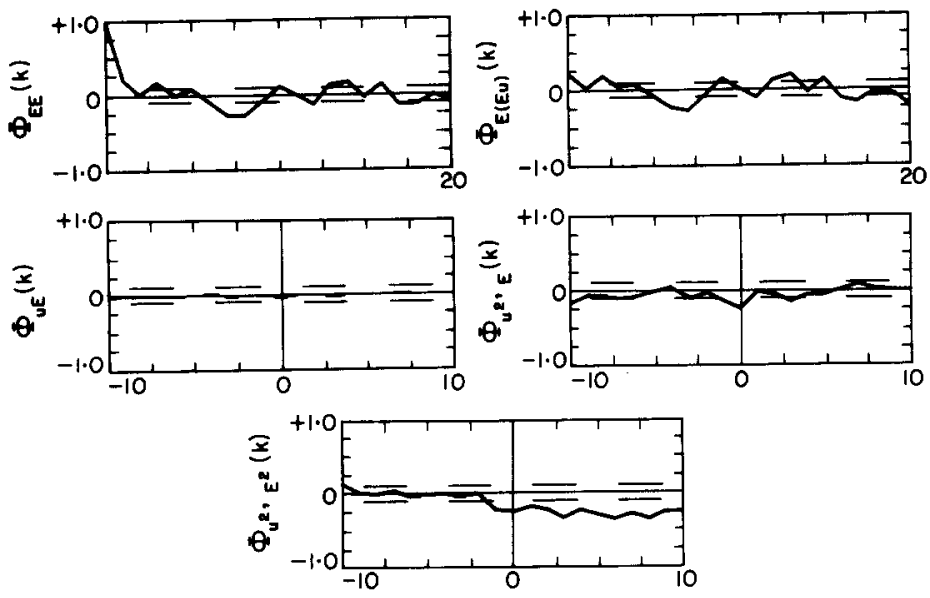

(a)
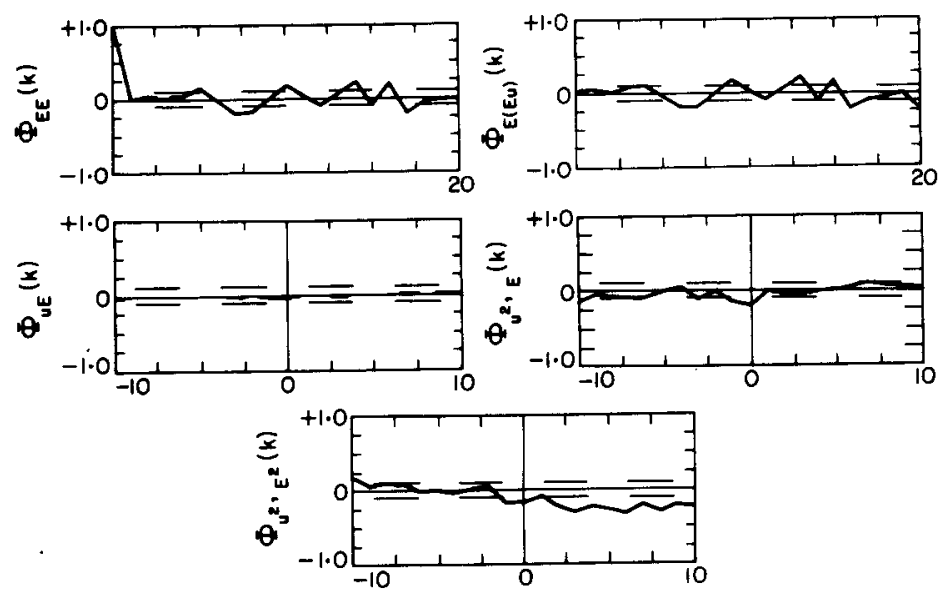

(b)
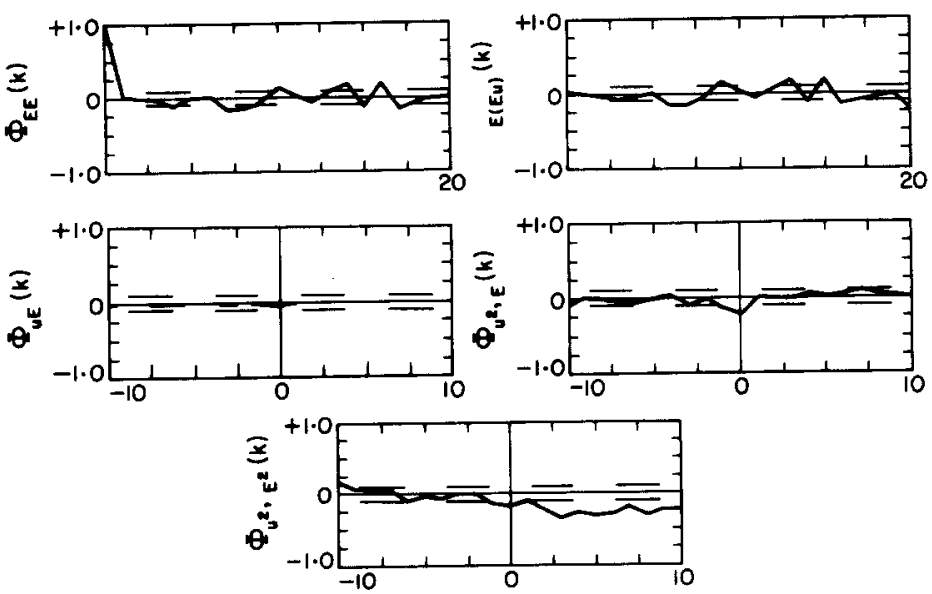

(c)

Figure 2. Correlation tests for linear model (mob6shv2.dta). (a) $n_{e}=0$; (b) $n_{e}=3$; (c) $n_{e}=5$. 
$n_{u}$ and $n_{e}$ were also fitted to the data but they did not improve $\Phi_{u^{2}{ }^{2}}(k)$. Consequently, the model

$$
\begin{aligned}
y(t)= & -0.34262+0 \cdot 74603 y(t-1)+0 \cdot 11912 u(t-1)+0 \cdot 15929 u(t-2)+0 \cdot 19844 e(t-1) \\
& +0.017201 e(t-2)+0.34711 e(t-3)+0 \cdot 26650 e(t-4)+0 \cdot 23640 e(t-5)+e(t)
\end{aligned}
$$

can be regarded as the best linear model. The predicted output and a chi-square test for model (24) are shown in Fig. 3. The best linear model of the engine for low engine speed conditions is therefore

$$
y(t)=-0 \cdot 34262+0 \cdot 74603 y(t-1)+0 \cdot 11912 u(t)+0 \cdot 15929 u(t-1) .
$$

It is important to emphasise that this model is biased $\left(\Phi_{u^{2}} \varepsilon^{2}(k) \neq 0\right)$ and therefore even though it provides what appears to be a good prediction of the true system output this
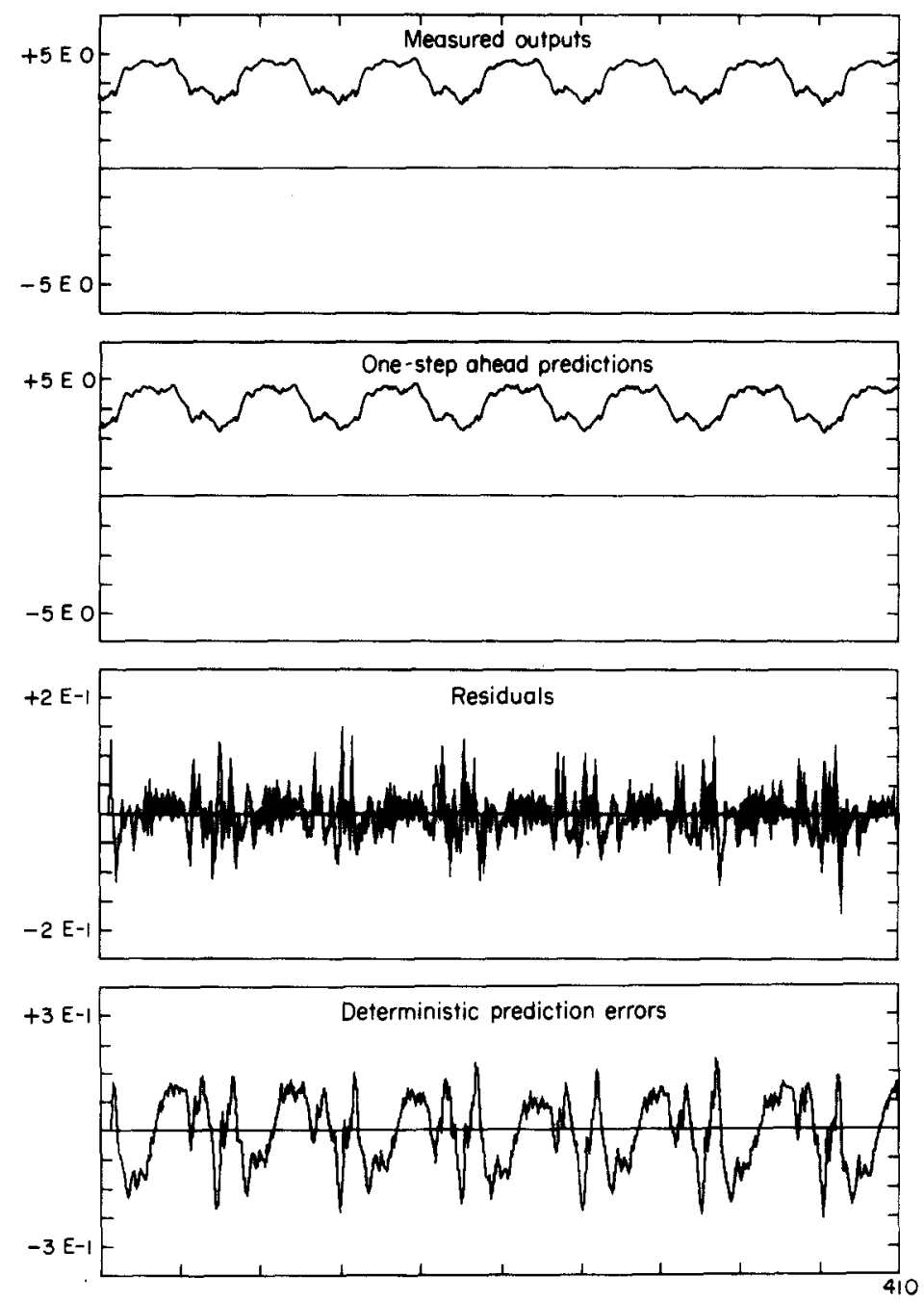

(a)

Figure 3. Best linear model for mob6shv2.dta. (a) $y(t), \hat{y}(t), \varepsilon(t)$ and $\varepsilon_{d}(t)$; (b) $\hat{y}_{d}(t)$ superimposed on $y(t)$. $\underset{ }{-}$, Model predicted outputs; - - , system outputs. (c) a chi-square test: $m(t)=\varepsilon(t-1) . \bullet \bullet, 95 \%$ confidence limit. 


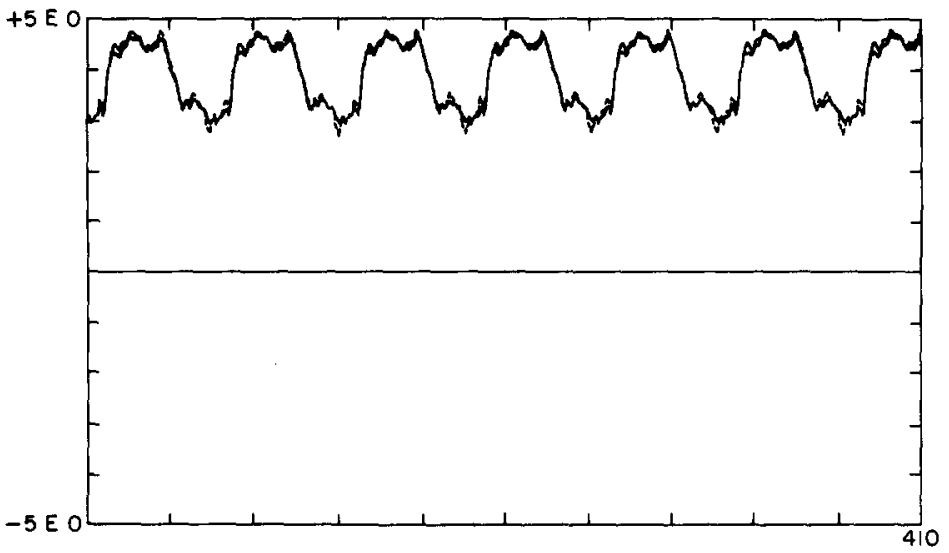

(b)

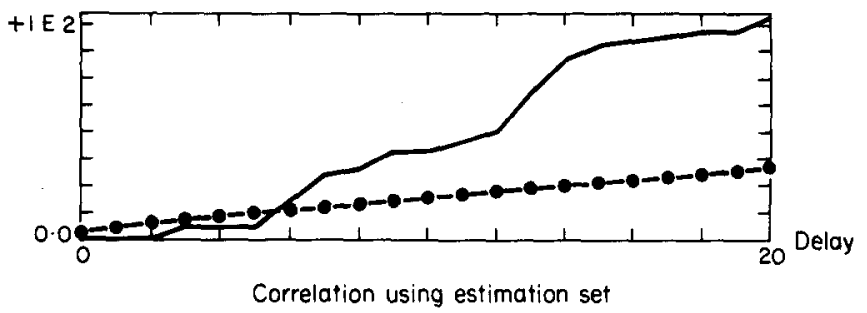

(c)

Figure 3-continued.

is no guarantee that a controller based on this model will perform adequately [2]. The bias means that the model is only valid for this particular data set and does not represent an adequate description of the underlying process which we seek. Figure 3(c) confirms the results of the correlation validity tests Fig. 2 and indicates that the residual sequence is correlated. Any normal linear analysis would terminate at this point because both $\Phi_{\varepsilon \varepsilon}(k)$ and $\Phi_{u \varepsilon}(k)$ are acceptable. However, as Fig. 2 clearly shows, $\Phi_{u^{2} \varepsilon^{2}}(k)$ is well outside the confidence bands indicating that significant non-linear terms have been omitted from the model. The effects of introducing non-linearities into the model are therefore investigated next.

\subsection{NON-LINEAR ANALYSIS OF THE LOW ENGINE SPEED CASE}

Non-linear terms were introduced into the model (23) and the prediction-error estimator was applied to the resulting models for different values of $n_{e}$. It was found that $n_{e}=5$ was appropriate. The prediction-error parameter estimates associated with this model are given in Table 4. The correlation tests, and the response of the model together with a chi-square test are illustrated in Figs 4 and 5 respectively.

A comparison of Fig. 3 with Fig. 5 shows that this non-linear model produces a better fit to the data than the best linear model (24). The variance of the residuals is almost one-third of that of the best linear model. $\Phi_{\varepsilon \varepsilon}(k)$ and $\Phi_{\varepsilon(\varepsilon u)}(k)$ are now well within the confidence limits, but $\Phi_{u^{2}}{ }^{2}{ }^{2}(k)$ which has improved slightly is still outside the confidence limits. The improvement in the residual sequence can be clearly seen in Fig. 5(c).

According to previous experience, the situation $\Phi_{u^{2}} e^{2}(k) \neq 0$ can be caused by omitting some internal noise terms $\beta u^{k}(\cdot) e^{i}(\cdot)$ from the model [8]. This suggests a way of improving the fitted model and bi-linear terms $y(\cdot) e(\cdot)$ and $u(\cdot) e(\cdot)$ were introduced 


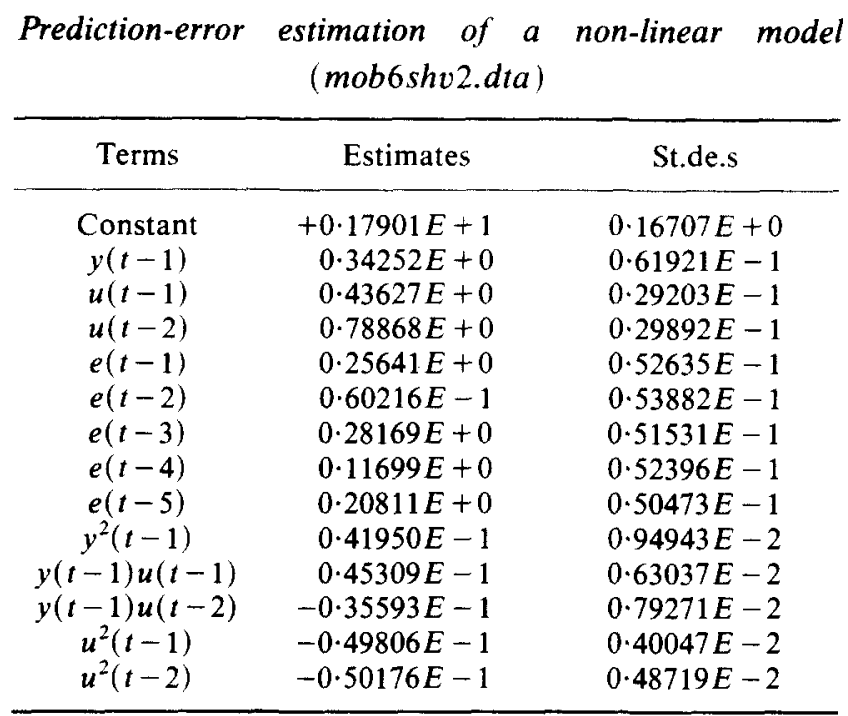

$\sigma_{\varepsilon}^{2}=0.73555 E-3$.
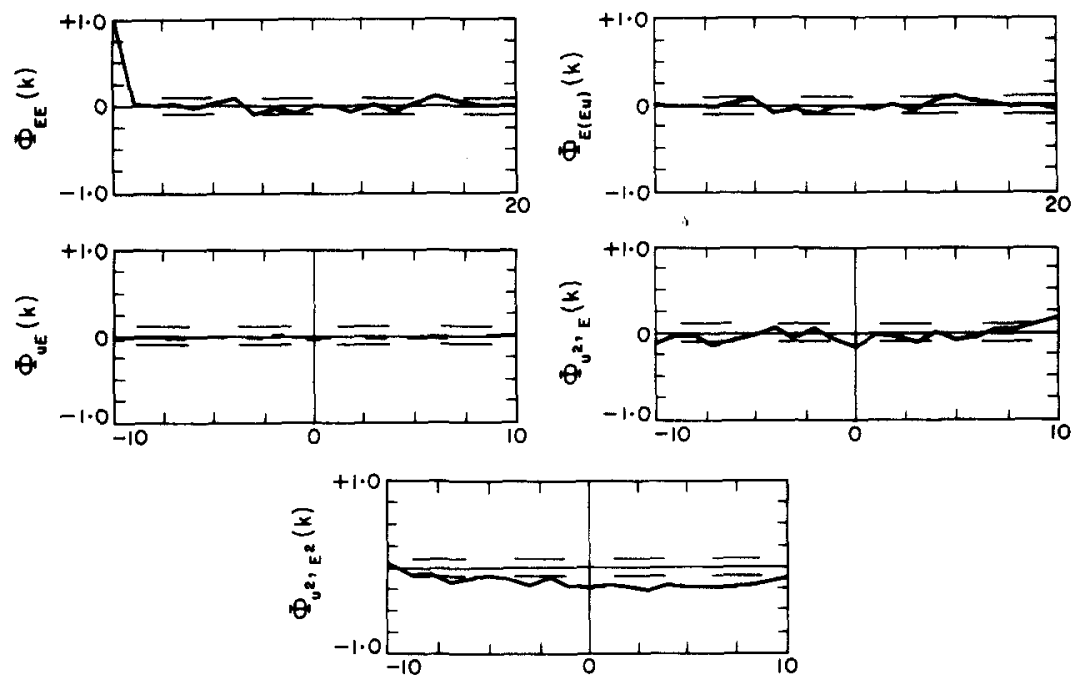

Figure 4. Correlation tests of the non-linear model given in Table 4.

into the model. The prediction-error estimator coupled with a model reduction routine $[11,16]$ produced the model in Table 5 . The improvement in $\Phi_{u^{2} \varepsilon^{2}}(k)$ can clearly be seen from Fig. 6.

Non-linear models with higher values of $n_{y}, n_{u}$ and $L$ were also investigated using the combined procedure of orthogonal and prediction-error estimation. In all cases the final models produced were more complex and gave similar model validation results. The model given in Table 5 was therefore accepted as the best non-linear model even though 

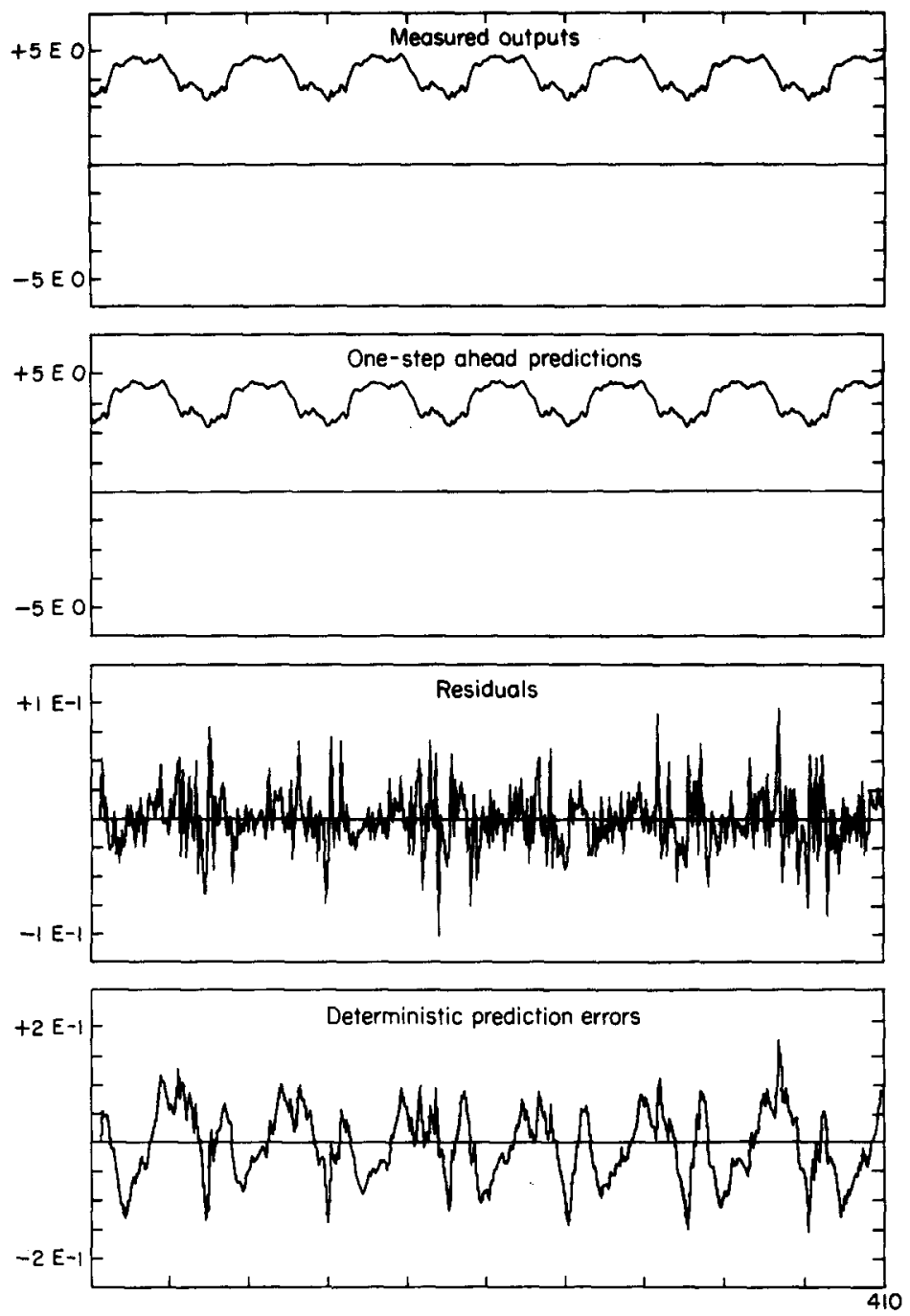

(a)

Figure 5. A non-linear model (Table 4) for mob6shv2.dta. (a) $y(t), \hat{y}(t), \varepsilon(t)$ and $\varepsilon_{d}(t) ;$ (b) $\hat{y}_{d}(t)$ superimposed on $y(t)$. - Model predicted outputs; - - , system outputs; (c) a chi-square test: $m(t)=\varepsilon(t-1) . \bullet-\bullet, 95 \%$ confidence limit.

$\Phi_{u^{z^{\prime}} \varepsilon^{2}}(k)$ was still slightly outside the confidence bands. We can only surmise that the complexity of the noise model in Table 5 and the difficulty with $\Phi_{u^{2} \varepsilon^{2}}(k)$ was induced by hand sampling the data, which may have introduced errors in the synchronisation of the input and output. The noise terms in Table 5 are only included to ensure that the estimates of the process model parameters are unbiased, and the best non-linear model for the engine under the experimental conditions considered is chosen as

$$
\begin{aligned}
y(t)= & -1.8647+0.25121 y(t-1)+0.49787 u(t)+0.83088 u(t-1)+0.055328 y^{2}(t-1) \\
& +0.054122 y(t-1) u(t)-0.050123 y(t-1) u(t-1) \\
& -0.058763 u^{2}(t)-0.04827 u^{2}(t-1)
\end{aligned}
$$




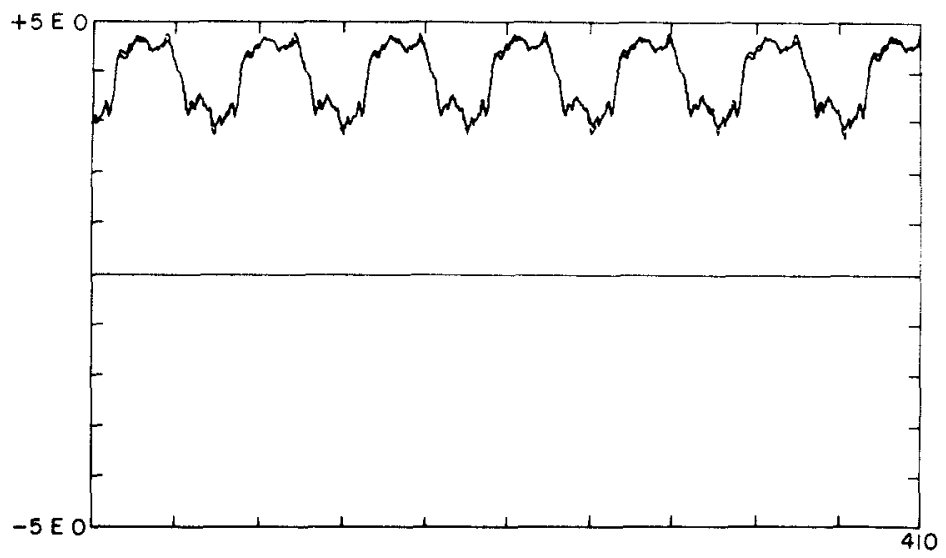

(b)

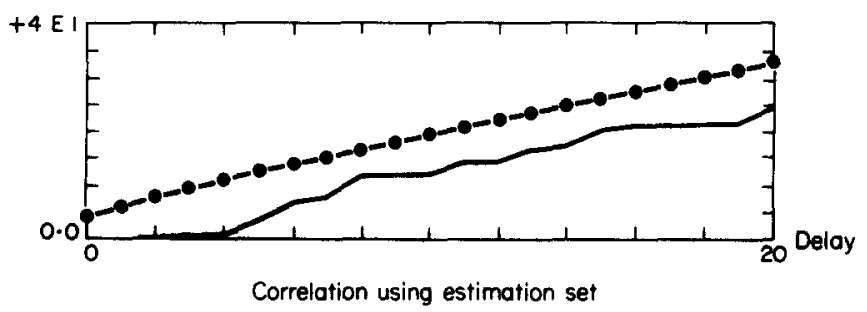

(c)

Figure 5-continued.

\subsection{LiNEAR ANALYSIS OF THE HIGH ENGINE SPEED CASE}

As in the case of mob6shv2.dta, the orthogonal estimator was initially used to determine the correct orders of the linear model. With a tolerance of 0.000009 , from the same 41 terms, the estimator selected the following nine terms (Table 6). The correlation tests shown in Fig. 7 indicate that this model is adequate. This together with the fact that the e.r.r. drops rapidly after the first four terms have been included seems to suggest that a linear model of the form

$$
y(t)=\text { constant }+a_{1} y(t-1)+a_{2} y(t-2)+b_{1} u(t-1)+b_{2} u(t-2)+e(t)
$$

is enough. Optimising the parameters in this model structure using a prediction-error estimator produced the results in Table 7. The correlation test plotted in Fig. 8 indicate that the model is unbiased even though the noise model order is zero. The excellent response of the model is illustrated in Fig. 9. Extensive chi-square tests also confirms that the model was indeed unbiased. Some of the chi-square tests are shown in Fig. 10.

Since the model validity tests clearly shows that in this case no non-linear terms should be included it was of interest to investigate if a model of the form of equation (23) could be used to describe the data set. The results showed that omitting the terms $a_{2} y(t-2)$ in equation (27) induced bias, and the best model for the engine under the high speed test conditions was therefore chosen as

$$
y(t)=-0 \cdot 25152+1 \cdot 0500 y(t-1)-0 \cdot 16765 y(t-2)+0 \cdot 11063 u(t)+0 \cdot 1073 u(t-1) .
$$


TABle 5

Prediction-error estimation of final non-linear model (mob6shv2.dta)

\begin{tabular}{crc}
\hline Terms & \multicolumn{1}{c}{ Estimates } & \multicolumn{1}{c}{ St.de.s } \\
\hline Constant & $-0 \cdot 18647 E+1$ & $0 \cdot 17102 E+0$ \\
$y(t-1)$ & $0 \cdot 25121 E+0$ & $0 \cdot 62218 E-1$ \\
$u(t-1)$ & $0 \cdot 49787 E+0$ & $0 \cdot 28157 E-1$ \\
$u(t-2)$ & $0 \cdot 83088 E+0$ & $0 \cdot 28628 E-1$ \\
$e(t-1)$ & $0 \cdot 26157 E+0$ & $0 \cdot 48272 E-1$ \\
$e(t-2)$ & $-0 \cdot 39234 E+0$ & $0 \cdot 32618 E+0$ \\
$e(t-3)$ & $0 \cdot 79580 E+0$ & $0 \cdot 32844 E+0$ \\
$e(t-4)$ & $0 \cdot 58926 E+0$ & $0 \cdot 31622 E+0$ \\
$e(t-5)$ & $0 \cdot 97346 E+0$ & $0 \cdot 30038 E+0$ \\
$y^{2}(t-1)$ & $0 \cdot 55328 E-1$ & $0 \cdot 10376 E-1$ \\
$y(t-1) u(t-1)$ & $0 \cdot 54122 E-1$ & $0 \cdot 61297 E-2$ \\
$y(t-1) u(t-2)$ & $-0 \cdot 50123 E-1$ & $0 \cdot 81499 E-2$ \\
$y(t-1) e(t-2)$ & $0 \cdot 50442 E+0$ & $0 \cdot 85041 E-1$ \\
$y(t-1) e(t-3)$ & $0 \cdot 61282 E+0$ & $0 \cdot 11357 E+0$ \\
$y(t-1) e(t-4)$ & $0 \cdot 60015 E+0$ & $0 \cdot 10916 E+0$ \\
$u^{2}(t-1)$ & $-0 \cdot 58763 E-1$ & $0 \cdot 36713 E-2$ \\
$u(t-1) e(t-2)$ & $-0.29549 E+0$ & $0 \cdot 81942 E-1$ \\
$u(t-1) e(t-3)$ & $-0 \cdot 33093 E+0$ & $0 \cdot 79550 E-1$ \\
$u(t-1) e(t-4)$ & $-0 \cdot 21994 E+0$ & $0.95213 E-1$ \\
$u(t-1) e(t-5)$ & $-0 \cdot 15602 E+0$ & $0 \cdot 63998 E-1$ \\
$u^{2}(t-2)$ & $-0 \cdot 48272 E-1$ & $0 \cdot 46949 E-2$ \\
$u(t-2) e(t-3)$ & $-0 \cdot 25665 E+0$ & $0 \cdot 95008 E-1$ \\
$u(t-2) e(t-4)$ & $-0 \cdot 33229 E+0$ & $0 \cdot 10032 E+0$ \\
\hline$\sigma_{\varepsilon}^{2}=0.63225 E-3$. & &
\end{tabular}
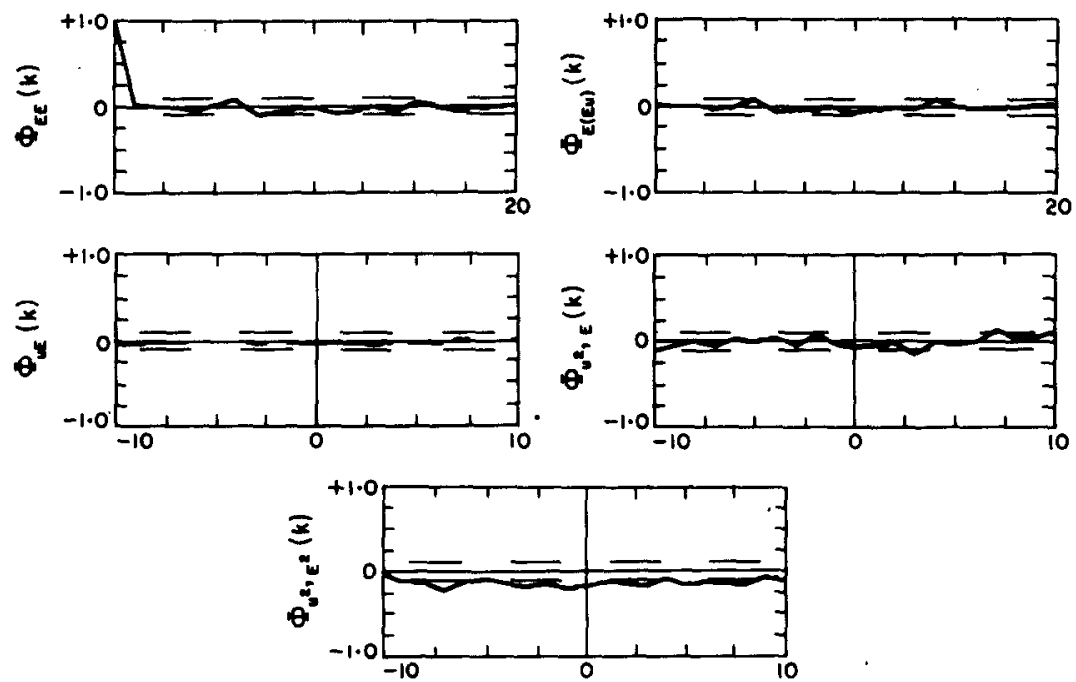

Figure 6. Correlation tests of the non-linear model given in Table 5. 
TABLE 6

Orthogonal estimation of linear model (mob6shv6.dta)

\begin{tabular}{ccrrr}
\hline O.s. & Terms & Estimates & \multicolumn{1}{c}{ E.r.r.s } & St.de.s \\
\hline 1 & $(2) y(t-1)$ & $0.9305 E+0$ & $0.9997 E+0$ & $0 \cdot 4839 E-1$ \\
2 & $(23) u(t-2)$ & $0 \cdot 1221 E+0$ & $0 \cdot 2121 E-3$ & $0.5871 E-2$ \\
3 & $(22) u(t-1)$ & $0 \cdot 1118 E+0$ & $0 \cdot 6753 E-4$ & $0 \cdot 2017 E-2$ \\
4 & $(1)$ constant & $-0 \cdot 3266 E+0$ & $0 \cdot 2567 E-4$ & $0 \cdot 2102 E-1$ \\
5 & $(3) y(t-2)$ & $-0 \cdot 7256 E-1$ & $0 \cdot 3540 E-5$ & $0 \cdot 4126 E-1$ \\
6 & $(12) y(t-11)$ & $0 \cdot 9775 E-2$ & $0 \cdot 4086 E-6$ & $0 \cdot 2118 E-2$ \\
7 & $(24) u(t-3)$ & $0 \cdot 1934 E-1$ & $0 \cdot 1103 E-6$ & $0 \cdot 6776 E-2$ \\
8 & $(25) u(t-4)$ & $0 \cdot 4775 E-2$ & $0 \cdot 8461 E-7$ & $0 \cdot 2888 E-2$ \\
9 & $(31) u(t-10)$ & $-0 \cdot 3339 E-2$ & $0 \cdot 5370 E-7$ & $0 \cdot 2202 E-2$ \\
\hline
\end{tabular}
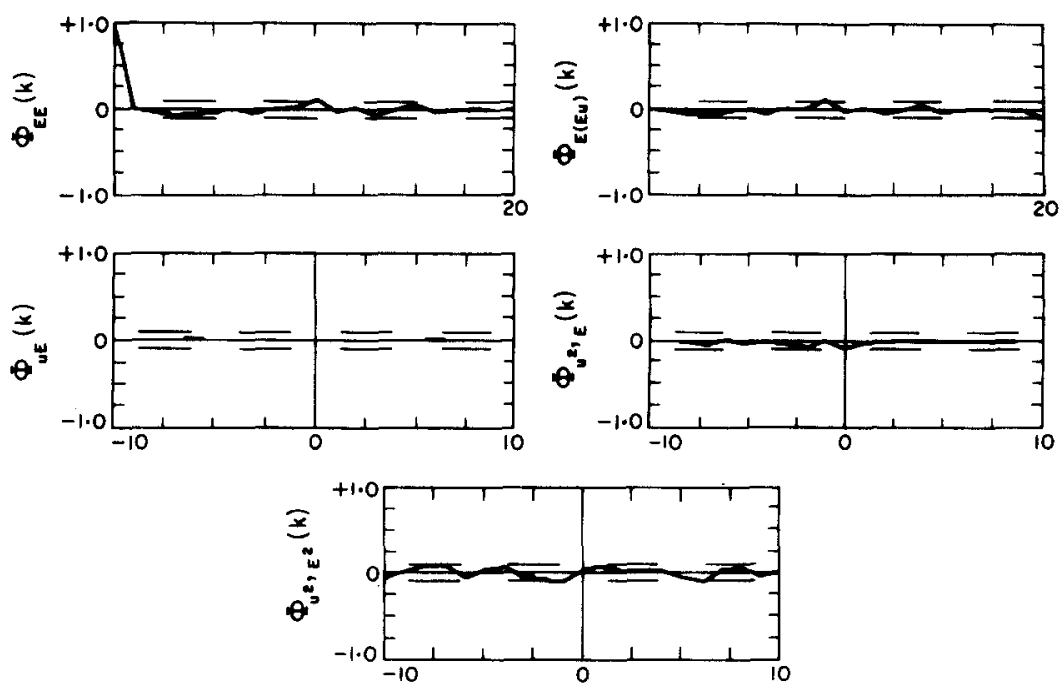

Figure 7. Correlation tests of the linear model given in Table 6.

TABLE 7

Prediction-error estimation of best linear model (mob6shv6.dta)

\begin{tabular}{crc}
\hline Terms & \multicolumn{1}{c}{ Estimates } & St.de.s \\
\hline Constant & $-0 \cdot 25152 E+0$ & $0 \cdot 10968 E-1$ \\
$y(t-1)$ & $0 \cdot 10500 E+1$ & $0 \cdot 15689 E-1$ \\
$y(t-2)$ & $-0 \cdot 16765 E+0$ & $0 \cdot 14440 E-1$ \\
$u(t-1)$ & $0 \cdot 11063 E+0$ & $0 \cdot 20673 E-2$ \\
$u(t-2)$ & $0 \cdot 10735 E+0$ & $0 \cdot 31474 E-2$ \\
\hline
\end{tabular}

$$
\sigma_{\varepsilon}^{2}=0 \cdot 44407 E-3
$$

\subsection{DISCUSSION OF RESULTS}

An automotive turbocharged diesel engine, other than one with very light boost, can be expected to become non-linear in its response to fuelling changes at the lower end of its normal speed range. This is because, even under steady state conditions, the boost 

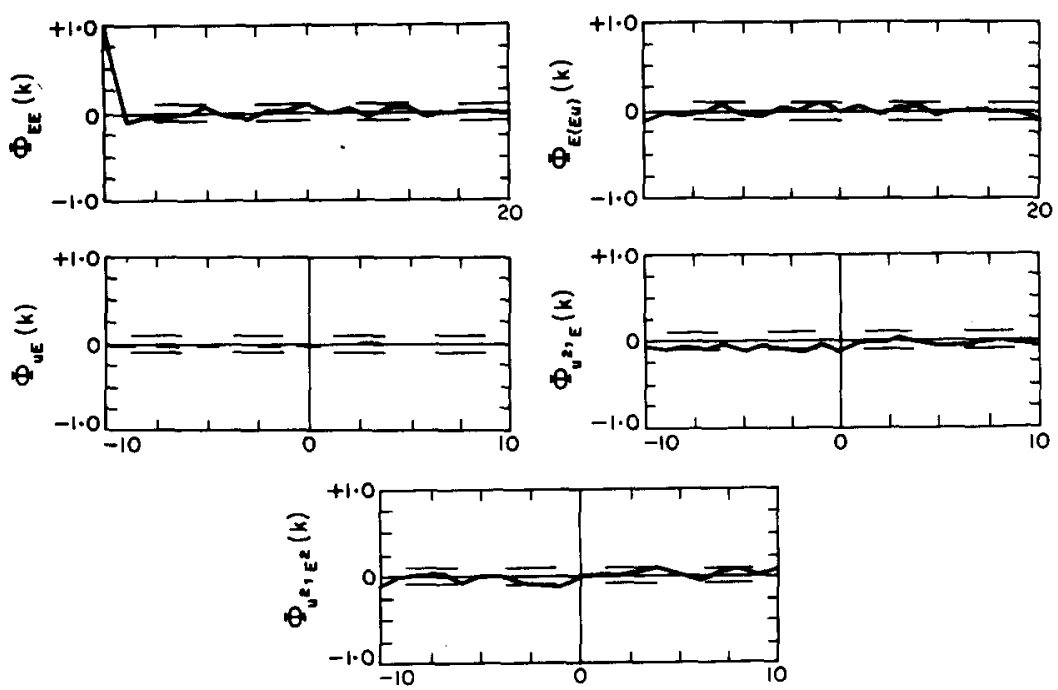

Figure 8. Correlation tests of best linear model (mob6shv6.dta).

pressure provided by the turbocharger gives an air-fuel ratio not much greater than that required for good combustion. The variation of torque with fuel injection rate becomes non-linear at lower air-fuel ratios and these occur during transients due to the well-known lag in response of the turbocharger. At higher engine speeds, the boost pressure provided by the turbocharger is sufficient to give a significant higher air-fuel ratio, so that saturation of the fuelling is much less likely to occur during transients. These expected characteristics are borne out by the identification results.

Engine speed response is of course dependent on the total load inertia, so that an identification of the speed response is only valid for one load condition (e.g. for one gear ratio and for one gross vehicle weight). The objective of the present study however was to demonstrate that parsimonious non-linear models can be used to predict engine response. The response of dynamic engine torque to fuelling is more difficult to determine [1]. The success of the NARMAX approach in the present study would however suggest that the latter problem would be a useful area to investigate in a future research study.

\section{CONCLUSIONS}

The identification of a turbocharged diesel engine has presented as an illustration of the practical application of an identification methodology for non-linear systems. It has been shown that the combined procedure of orthogonal and prediction-error estimation coupled with correlation and chi-squared model validity tests provides a powerful interactive toolkit for fitting parsimonious models to practical systems.

\section{ACKNOWLEDGEMENTS}

This work is partly supported by the Science and Engineering Research Council (Grant Ref. GR/D/30587). Richard Backhouse acknowledges the cooperation of Professor Wallace and the staff of the Wolfson Engine Test Unit at Bath University. 

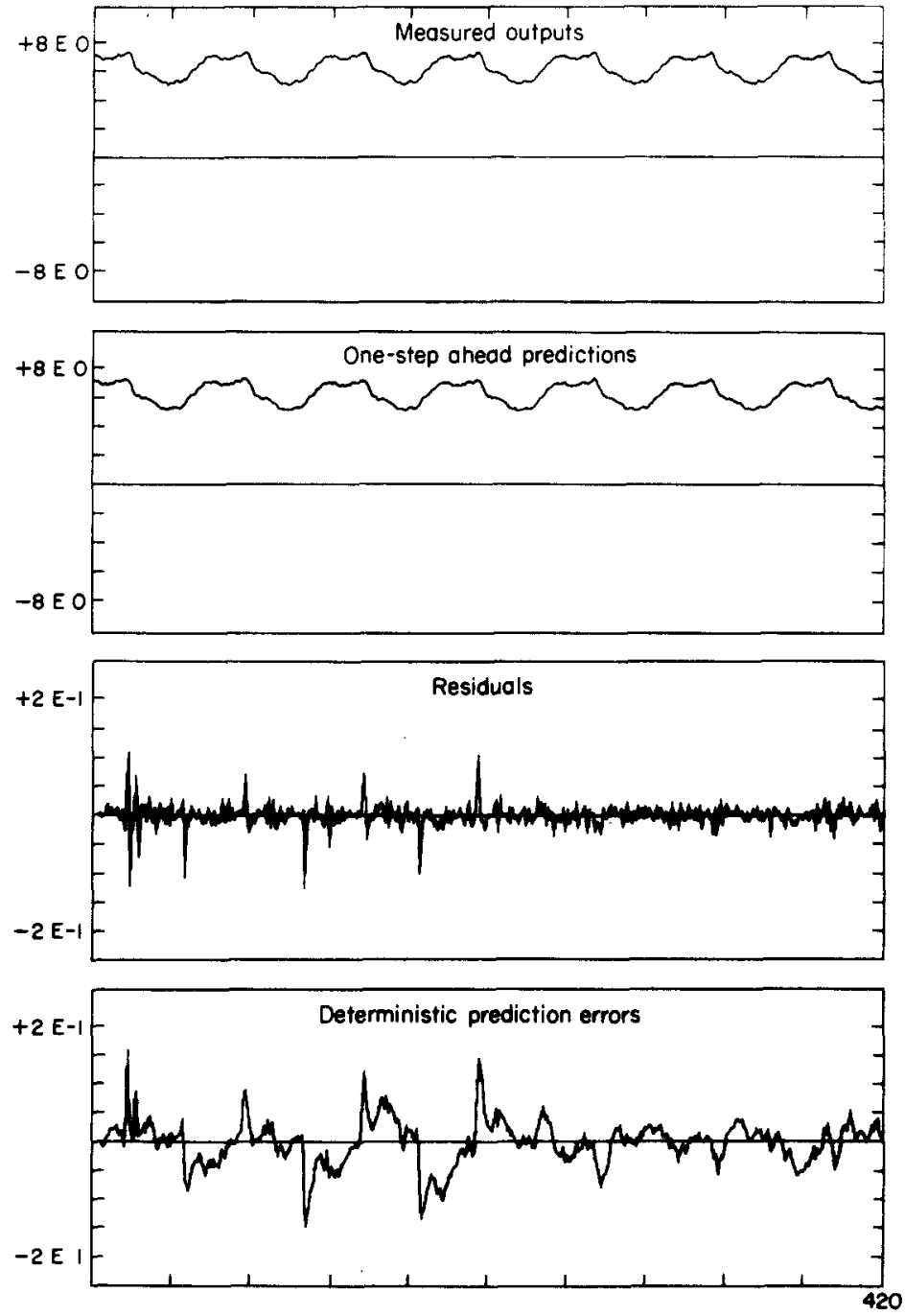

(a)

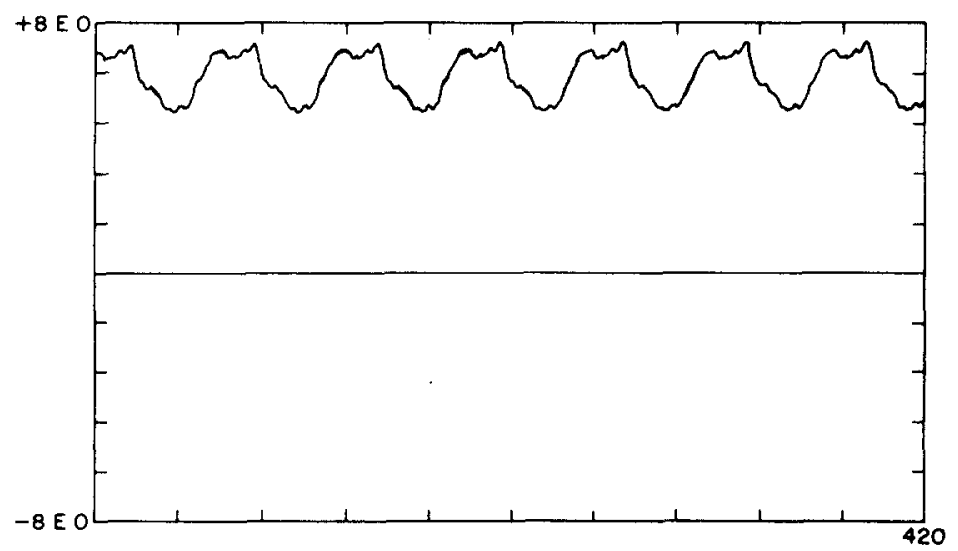

(b)

Figure 9. Best linear model for mob6shv6.dta. (a) $y(t), \hat{y}(t), \varepsilon(t)$ and $\varepsilon_{d}(t) ;(b) \hat{y}_{d}(t)$ superimposed on $y(t)$. Model predicted outputs; - - -, system outputs. 

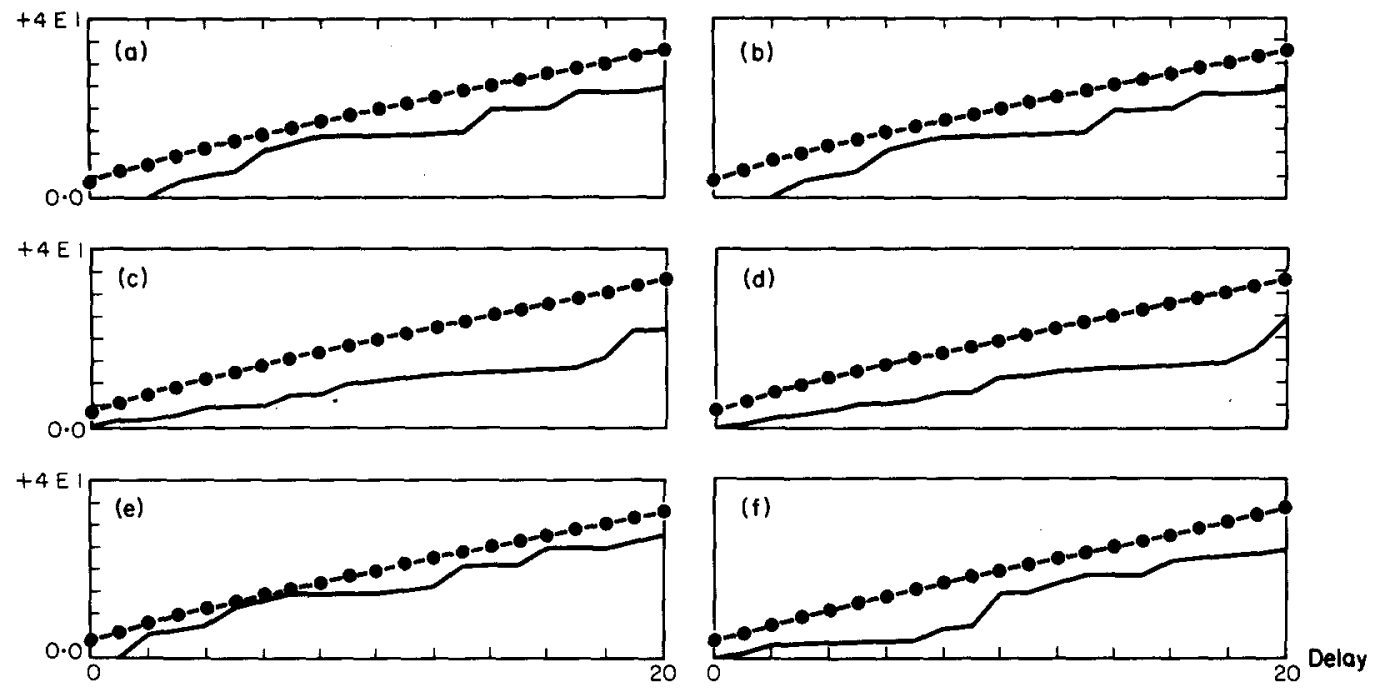

Correlation using estimation set

Figure 10. Some chi-square tests for best linear model (mob6shv6.dta). $\bullet-\bullet, 95 \%$ confidence limit. (a) $m(t)=y(t-1)$. (b) $m(t)=u(t-2) ;$ (c) $m(t)=\varepsilon(t-3) ;$ (d) $m(t)=y^{2}(t-1) \varepsilon(t-2) ;\left(\right.$ e) $m(t)=y^{2}(t-2)$; (f) $m(t)=u^{2}(t-3)$.

\section{REFERENCES}

1. R. J. BACKHOUSE and D. E. WINTERBONE 1986 SAE International Congress and Exposition, Detroit, Michigan, Feb. 1986 (SAE paper 860453). Dynamic behaviour of a turbocharged diesel engine.

2. S. A. Billings 1986 Signal Processing for Control in (K. Godfrey and P. Jones, editor) 261-294. Introduction to nonlinear systems analysis and identification. New York: Springer.

3. S. A. Billings and S. CHEN 1988 International Journal of Systems Science Identification of nonlinear rational systems using a prediction-error estimation algorithm (to appear).

4. S. A. BILlingS and M. B. FADZIL 1985 Proceedings of the 7 th IFAC Symposium on Identification and System Parameter Estimation, York, U.K., 155-160. The practical identification of systems with nonlinearities.

5. S. A. BILlings, M. B. FADzil, J. SUlley and P. M. Johnson 1988 Mechanical Systems and Signal Processing 2, 59-76. Identification of a non-linear difference equation model of an industrial diesel generator.

6. S. A. BILlings, M. J. KoRENBERG and S. CHEN 1988 International Journal of Systems Science 19, 1559-1568. Identification of non-linear output-affine systems using an orthogonal least squares algorithm.

7. S. A. Billings and I. J. LeONTARITIS 1981 Proceedings of IEE Conference on Control and its Applications, Warwick, U.K., 183-187. Identification of nonlinear systems using parameter estimation techniques.

8. S. A. BILLINGS and W. S. F. VOON 1986 International Journal of Control 44, 235-244. Correlation based model validity tests for non-linear models.

9. S. A. Billings and W. S. F. Voon 1986 International Journal of Control 44, 803-822. A prediction-error and stepwise-regression estimation algorithm for non-linear systems.

10. T. Bohlin 1978 Automatica 14, 137-146. Maximum-power validation of models without higher-order fittings.

11. S. CheN and S. A. Billings 1988 International Journal of Control 47, 309-332. Prediction-error estimation algorithm for non-linear output-affine systems.

12. M. J. Korenberg, S. A. Billings, Y. P. LiU and P. J. MCIlroy 1988 International Journal of Control, 48, 193-210. Orthogonal parameter estimation algorithm for non-linear stochastic systems. 
13. I. J. LeONTARITIS and S. A. BILlings 1985 International Journal of Control 41, 303-344. Input-output parametric models for non-linear systems, Part I: deterministic non-linear systems; Part II: stochastic non-linear systems.

14. I. J. LEONTARITIS and S. A. Billings 1987 International Journal of Control 45, 311-341. Model selection and validation methods for non-linear systems.

15. I. J. LeONTARITIS and S. A. BILlings 1987 International Journal of Systems Science 18, 189-202. Experimental design and identifiability for non-linear systems.

16. 1. J. LeONTARITIS and S. A. Billings 1988 International Journal of Systems Science 19, 519-536. Prediction error estimator for non-linear stochastic systems.

17. Y. P. LiU, M. J. KORENBERG, S. A. BILlings and M. B. FAZDil 1987 Presented at the 26th IEEE Conference on Decision and Control, Dec 9-11, 1987, Los Angeles. The nonlinear identification of a heat exchanger. 